
полисегментарной пневмонии

\author{
О.И.Савушкина ${ }^{1}$, А.В.Черняк², Н.Ю.Коповая ${ }^{1}$ \\ 1 - ФГКУ «Главный военный клинический госпиталь имени Н.Н. Бурденко» Минобороны России: 105229, Москва, Госпитальная пл., 3 ; \\ 2 - ФГБУ «НИИ пульмонологии» ФМБА России: 105077, Москва, ул. 11-я Парковая, 32, корп. 4
}

Резюме

Целью исследования явился анализ показателей комплексного исследования функции внешнего дыхания (ФВД) (спирометрия, бодиплетизмография и диффузионная способность легких - ДСЛ) у больных после перенесенной полисегментарной внебольничной пневмонии (ВП). Материалы и методы. Все анализируемые показатели ФВД получены с соблюдением стандартов исследования Европейского респираторного общества и Американского торакального общества (2005). ДСЛ (трансфер-фактор) оценивалась по оксиду углерода методом однократного вдоха с задержкой дыхания. Полученный показатель корригировался по уровню гемоглобина. Результаты. Показано, что полное клиническое и рентгенологическое разрешение ВП не всегда сопровождается нормализацией функции легких. Заключение. Нормализация показателей вентиляции и легочного газообмена является важным критерием полноты выздоровления, сроков восстановления трудоспособности и определяет показания для последующей диспансеризации больных.

Ключевые слова: внебольничная пневмония, вентиляционные нарушения, диффузионная способность легких.

DOI: 10.18093/0869-0189-2016-26-2-186-189

\title{
A role of pulmonary function testing for detecting ventilation and gas exchange disorders in multilobar community-acquired pneumonia convalescents
}

\author{
O.I.Savushkina ${ }^{1}$, A.V.Chernyak ${ }^{2}$, N.Yu.Kopovaya ${ }^{1}$ \\ 1 - Acad. N.N.Burdenko The Main Military Clinical Hospital, Moscow, Russia: 3, Gospital'naya sqr., Moscow, 105229, Russia; \\ 2 - Federal Pulmonology Research Institute, Federal Medical and Biological Agency of Russia: 32, build. 4, 11 ${ }^{\text {th }}$ Parkovaya str., Moscow, 105077, Russia
}

\section{Summary}

The aim of this study was to analyze lung function in multilobar community-acquired pneumonia (CAP) convalescents. Methods. Spirometry, body plethysmography, and lung diffusing capacity were measured according to the joint European Respiratory Society and American Thoracic Society standards (2005). The carbon monoxide lung diffusing capacity was evaluated using a single breath-hold technique and was adjusted for hemoglobin. Results. The complete clinical and radiological resolution of CAP did not accompanied by recovery of lung function in all cases. Obstructive ventilation disorders were diagnosed in $18.2 \%$ of patients, restrictive ventilation disorders were diagnosed in $12.1 \%$ of patients, and gas exchange abnormalities were diagnosed in $51.5 \%$ of the patients. Conclusion. Lung function and gas exchange testing is an important marker of the patient's convalescence after CAP and duration of the recovery; it also could predict the need in subsequent follow-up.

Key words: community-acquired pneumonia, ventilation disorders, lung diffusing capacity.

Проблема внебольничной пневмонии (ВП) остается актуальной в мировом масштабе. По данным статистики, заболеваемость ВП составляет 10-12 случаев на 1000 (10-12\%o) населения. В России общее число больных ВП ежегодно превышает 1,5 млн человек, при этом наиболее высокий уровень смертности традиционно регистрируется среди мужчин трудоспособного возраста [1].

Болезни органов дыхания (БОД) занимают лидирующие позиции в структуре патологии внутренних органов среди молодых лиц из организованных коллективов. В 2012 г. 54 \% всех заболеваний военнослужащих по призыву пришлись на острые БОД: острые респираторные заболевания, тонзиллит, бронхит и пневмонии. ВП среди них диагностирована в $13 \%$ случаев (49 на 1000 (49 \%о) военнослужащих), что значительно превосходит тот же показатель в общей популяции [2]. В структуре первичной заболеваемости среди военнослужащих по призыву БОД, в т. ч. ВП, в 2013 г. составили $62 \%$ [3]. В ряде случаев не удалось избежать тяжелого течения ВП [4, 5].

Пневмония - инфекция дистальных отделов респираторного тракта, характеризующаяся вовлечением в патологический процесс альвеол, бронхов мелкого калибра и бронхиол. ВП - острое заболевание, возникшее во внебольничных условиях и сопровождающееся симптомами инфекции нижних отделов дыхательных путей (лихорадкой, кашлем, выделением мокроты, болью в грудной клетке, одышкой) и рентгенологическими признаками свежих очагово-инфильтративных изменений в легких при отсутствии очевидной диагностической альтернативы. У большинства больных ВП через 3-5 дней эффективной антибактериальной терапии клинические 
проявления заболевания регрессируют. При этом рентгенологическое выздоровление, как правило, отстает от клинического. В случаях, когда на фоне улучшения клинической картины к исходу 4-й недели от начала заболевания не удается достичь полного рентгенологического разрешения очаговоинфильтративных изменений в легких, следует говорить о затяжной ВП [1]. При этом полное клиническое и рентгенологическое разрешение ВП не всегда сопровождается нормализацией функции легких, для исследования которой чаще всего прибегают к спирометрии. При проведении спирометрии с регистрацией кривой поток / объем форсированного выдоха прежде всего выявляются обструктивные нарушения вентиляционной функции, что немаловажно для больных, перенесших острую инфекцию нижних отделов дыхательных путей. Однако нормальные показатели спирометрии не всегда позволяют судить о полном восстановлении функции внешнего дыхания (ФВД), включающей не только вентиляцию, но и газообмен. Изучение легочного газообмена (измерение диффузионной способности легких - ДСЛ) является важным этапом в выявлении нарушения респираторной функции, т. к. позволяет оценить тяжесть патологических изменений легочной ткани. Кроме того, исследование ДСЛ имеет прогностическое значение в отношении течения заболевания и играет важную роль в оценке эффективности лечения. При некоторых заболеваниях легочной паренхимы изменение ДСЛ может быть единственным функциональным нарушением, выявленным у больного. Так, у больных с интерстициальными заболеваниями легких снижение диффузии может определяться в > 70 \% случаев, тогда как снижение жизненной емкости легких (ЖЕЛ) в $50 \%$, а снижение общей емкости легких (ОЕЛ) в $<20 \%$ случаев [6]. Следовательно, изучение ДСЛ, зависящей от структурных и функциональных свойств легочной ткани, имеет большое диагностическое значение при паренхиматозных заболеваниях легких, в т. ч. при пневмониях. Однако публикаций, посвященных изучению ФВД после перенесенной пневмонии, недостаточно.

Целью данной работы явилось выявление вентиляционно-газообменных нарушений у лиц молодого возраста из организованных коллективов после перенесенной полисегментарной ВП для разработки индивидуальных программ реабилитации на госпитальном и постгоспитальном этапах.

\section{Материалы и методы}

В исследование включены пациенты (мужчины) в возрасте от 18 до 40 лет $(n=33$; средний возраст $23,78 \pm 5,58$ года) после перенесенной полисегментарной ВП. У 4 (12,1 \%) пациентов исходом была адгезия костальной плевры, еще у 4 (12,1\%) - пневмофиброз, у $3(9,0 \%)$ - как реакция плевры, так и пневмофиброз.

В работе использованы современные методы исследования ФВД - спирометрия, бодиплетизмогра- фия, диффузионный тест, которые проводились на установке Master Screen Body и Master Screen Diffusion (CareFusion (Jaeger), Германия) с соблюдением стандартов исследования American Thoracic Society (ATS) и European Respiratory Society (ERS) (2005) [7-9]. ДСЛ оценивалась по оксиду углерода методом однократного вдоха с задержкой дыхания и коррекцией полученных данных по уровню гемоглобина. Проанализированы следующие показатели:

- спирометрические: форсированная ЖЕЛ (ФЖЕЛ); объем форсированного выдоха за 1-ю секунду $\left(\mathrm{OФB}_{1}\right)$; отношение ОФВ 1 / ЖЕЛ после регистрации ЖЕЛ; ОФВ 1 / ФЖЕЛ; средняя объемная скорость на среднем участке кривой поток / объем форсированного выдоха между 25 и $75 \%$ ФЖЕЛ $\left(\mathrm{COC}_{25-75 \%)}\right.$;

- статические легочные объемы и емкости: ОЕЛ, ЖЕЛ и составляющие ее объемы емкости вдоха $\left(\mathrm{E}_{\text {вд. }}\right)$ и резервный объем выдоха $\left(\mathrm{PO}_{\text {выд. }}\right)$; остаточный объем легких (ООЛ); отношение ООЛ / ОЕЛ; внутригрудной объем газа (ВГО);

- показатель диффузии СО (трансфер-фактор $\mathrm{DL}_{\mathrm{CO}}$ ) и отношение $\mathrm{DL}_{\mathrm{co}}$ к объему альвеолярной вентиляции $\left(\mathrm{V}_{\mathrm{A}}\right)$.

Оценка величин вентиляционных показателей и степень выраженности их изменений проводилась с учетом требований ERS и ATS (2005) [7], а также Руководств под редакцией Л.Л.Шика, Н.Н.Канаева [10] и М.М.Ильковича, А.Н.Кокосова [11].

Степень тяжести снижения ДСЛ определялась согласно градации (табл. 1).

Для молодых людей в возрасте 25 лет НГН составляет 80 \% долж., тогда как для людей более старшего возраста НГН может оказаться $<80$ \% дол. Следовательно, необходимо оценивать попадание фактических значений (мл / мин / мм рт. ст. или моль / мин / кПа) в диапазон значений нижней и верхней границ нормы, величину которых современные программы позволяют выводить на монитор.

Статистическая обработка результатов проведена методами описательной статистики с применением прикладного пакета программ Statistica 6.0. После проверки нормальности распределения показателей по критерию Стьюдента были рассчитаны коэффициенты ранговой корреляции Спирмена с целью оценки взаимосвязи пневмофиброза и реакции плевры с анализируемыми показателями ФВД. Величина уровня статистической значимости принята равной 0,05 .

Таблица 1 Степени тяжести снижения ДСЛ [7] Table 1 Severity of decrease in lung diffusing capacity for carbon monoxide [7]

\begin{tabular}{|l|c|}
\hline Степень тяжести снижения $\mathrm{DL}_{\mathrm{co}}$ & $\mathrm{DL}_{\mathrm{co}}, \%_{\text {долж. }}$ \\
\hline Легкая & $>60 \%$ и $<$ HгH \\
\hline Умеренная & $40-60$ \\
\hline Тяжелая & $<40$ \\
\hline
\end{tabular}

Примечание: НГН - нижняя граница нормы (мл / мин / мм рт. ст. или моль / мин / КПа). 


\section{Результаты и обсуждение}

У 23 (69,7 \%) пациентов показатели спирометрии соответствовали возрастной норме. Снижение ЖЕЛ зарегистрировано у $4(12,1 \%)$ больных: у 3 - за счет снижения $\mathrm{E}_{\text {вд. }}$ у 1 - за счет снижения как $\mathrm{E}_{\text {вд., так }}$

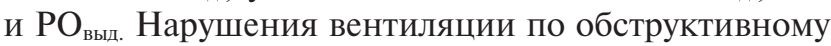
типу выявлены у $6(18,2 \%)$ пациентов, у всех - легкая степень обструкции.

По данным бодиплетизмографии показано снижение ОЕЛ у 4 (12,1\%) пациентов, что позволило выявить нарушения легочной вентиляции по рестриктивному типу. С учетом того, что у данных пациентов показатель ОФВ 1 превышал 70 \% долж., согласно рекомендациям ERS и ATS (2005) [7] диагностировано нарушение легкой степени. Уменьшение ОЕЛ у 1 больного сопровождалось понижением ЖЕЛ, еще у 1 - понижением ЖЕЛ и ООЛ, тогда как в 2 случаях ОЕЛ была понижена только за счет уменьшения ООЛ. Кроме того, снижение ООЛ, не сопровождавшееся снижением ОЕЛ и других составляющих ее объемов, выявлено еще у 8 (24,2 \%) пациентов. Как известно, снижение ООЛ иногда является единственным физиологическим отклонением вентиляционно-газообменной функции при некоторых состояниях, в т. ч. у пациентов с инфекционными заболеваниями [12], и может служить маркером увеличения эластической тяги легочной ткани, т. е. снижения ее статической растяжимости [10].

Увеличение ООЛ обнаружено у 3 (9 \%) пациентов, которое только в 1 случае сопровождалось снижением индекса Тиффно и повышением показателя ООЛ / ОЕЛ, что позволило диагностировать наличие «воздушных ловушек». Как известно, нормальные данные спирометрии и повышение ООЛ [13], а также сочетание повышенного ООЛ с нормальным бронхиальным сопротивлением на выдохе [14] указывают на изолированную обструкцию мелких ( $<2$ мм в диаметре) бронхов, т. е. на изолированную дистальную обструкцию, которая и была диагностирована у 2 (6\%) пациентов.

У $17(51,5 \%)$ пациентов отмечалось снижение показателя $\mathrm{DL}_{\mathrm{co}}$ легкой степени, тогда как значение $\mathrm{DL}_{\mathrm{co}} / \mathrm{V}_{\mathrm{A}}$ во всех случаях оставалось в норме. У $11(64,7 \%)$ пациентов с пониженным показателем $\mathrm{DL}_{\mathrm{co}}$ не выявлено нарушений вентиляционной функции легких; у 4 (23,5 \%) установлен рестриктивный тип нарушения легочной вентиляции, у $2(11,8 \%)$ - обструктивный тип нарушения легочной вентиляции.

Таким образом, при комплексном исследовании ФВД после перенесенной полисегментарной ВП у 18,2 \% пациентов выявлено обструктивное нарушение легочной вентиляции; у 12,1 \% - рестриктив- ное нарушение легочной вентиляции; у 51,5 \% - нарушение газообменной функции легких.

У 2 (8,6\%) из $23(69,7 \%)$ пациентов с нормальными показателями спирометрии при выполнении бодиплетизмографии и диффузионного теста выявлена изолированная дистальная обструкция (увеличение ООЛ); у $11(47,8 \%)$ - нарушение ДСЛ (снижение показателя $\left.\mathrm{DL}_{\mathrm{co}}\right)$; у $8(34,7$ \%) - снижение ООЛ, которое у 2 больных обусловило снижение ОЕЛ, что, в свою очередь, несмотря на нормальные данные спирометрии, дало возможность диагностировать рестриктивное нарушение легочной вентиляции. Следовательно, с целью выявления вентияционногазообменных нарушений после перенесенной полисегментарной ВП спирометрию следует дополнять бодиплетизмографией и исследованием ДСЛ.

Результаты описательной статистики перечисленных показателей комплексного исследования ФВД представлены в табл. 2 в формате среднее значение \pm ошибка среднего $(M \pm m)$.

Расчет коэффициентов ранговой корреляции Спирмена у пациентов с остаточным пневмофиброзом выявил слабую обратную корреляционную связь с большинством показателей вентиляции и газообмена, за исключением ООЛ ( $\rho=-0,36 ; p<0,036)$, ООЛ / ОЕЛ ( $\rho=-0,42 ; p<0,013)$ и $\operatorname{DL}_{\text {Со }}(\rho=-0,34$; $p<0,048)$, с которыми установлена средняя обратная корреляционная связь. Полученные данные позволяют судить о том, что снижение ООЛ и ООЛ / ОЕЛ в ряде случаев может служить маркером изменения эластических свойств легочной ткани, обусловленных пневмофиброзом, и согласуется с данными, полученными М.Ю.Каменевой и соавт. [15]. Кроме того, снижение показателя $\mathrm{DL}_{\mathrm{co}}$, свидетельствующее о нарушении ДСЛ, позволяет судить об остаточных поствоспалительных изменениях в легких. Таким образом, понижение показателей ООЛ, ООЛ / OЕЛ и DL жет являться функциональным признаком изменения структурных свойств легочной ткани.

При расчете коэффициентов ранговой корреляции Спирмена у пациентов с реакцией плевры не выявлено достоверного влияния перенесенного плеврита на вентиляционно-газообменную функцию легких.

\section{Заключение}

Полное клиническое и рентгенологическое разрешение ВП не всегда сопровождается нормализацией функции легких. Снижение ДСЛ является наиболее частым (51,5 \% случаев) нарушением ФВД после перенесенной ВП, тогда как различные типы нарушений легочной вентиляции встречаются у $30,3 \%$ реконвалесцентов.

Таблица 2

Средние значения показателей комплексного исследования ФВД в изученной выборке Table 2

Mean values of lung function parameters in patients

\begin{tabular}{|c|c|c|c|c|c|c|c|c|c|c|c|c|}
\hline Показатель & ЖЕЛ & ФЖЕЛ & $0 Ф \mathrm{~B}_{1}$ & $\begin{array}{c}\text { OФB }_{1} / \\
\text { ЖЕЛ }\end{array}$ & $\begin{array}{l}\text { ОФВ } 1 / \\
\text { ФЖЕЛ }\end{array}$ & $\operatorname{COC}_{25-75 \%}$ & ОЕЛ & оол & $\mathrm{PO}_{\text {выд. }}$ & $\mathrm{E}_{\mathrm{Bд}}$ & $\mathrm{DL}_{c 0}$ & $\mathrm{DL}_{\mathrm{co}} / \mathrm{VA}$ \\
\hline$M \pm m$ & $96,1 \pm 2,1$ & $96,1 \pm 1,8$ & $96,2 \pm 2,2$ & $80,9 \pm 1,5$ & $83,1 \pm 1,1$ & $85,7 \pm 3,4$ & $95,9 \pm 2,1$ & $97,2 \pm 4,8$ & $95,7 \pm 3,8$ & $94,0 \pm 3,1$ & $81,3 \pm 2,1$ & $103,4 \pm 2,0$ \\
\hline
\end{tabular}

Примечание: $M \pm m$ - среднее значение \pm стандартное отклонение. 
Таким образом, нормализация показателей вентиляции и легочного газообмена является важным критерием полноты выздоровления, сроков восстановления трудоспособности и определяет показания для последующей диспансеризации больных. Следовательно, у лиц, перенесших ВП с остаточными вентиляционно-газообменными нарушениями, целесообразно использовать:

- бронхорасширяющие препараты при терапии;

- различные методики дыхательной гимнастики при более длительном периоде реабилитации;

- мониторинг показателей ФВД до их восстановления.

Конфликт интересов отсутствует.

Исследование проводилось без участия спонсоров.

There is no conflict of interest.

The study was performed without any sponsorship.

\section{Литература}

1. Чучалин А.Г., ред. Пульмонология: Национальное руководство. М.: ГЭОТАР-Медиа; 2013.

2. Жоголев С.Д., Огарков П.И., Жоголев К.Д. и др. Эпидемиология и профилактика внебольничных пневмоний у военнослужащих. Военно-медицинский журнал. 2013; 334 (11): 55-60.

3. Калмыков А.А., Носарев В.Г., Аминев Р.М., Коновалов П.П. Роль взаимодействия командования и медицинской службы округа в профилактике острых болезней органов дыхания. Военно-медицинский журнал. 2014; 335 (11): 11-16.

4. Овчинников Ю.В., Азаров И.И., Кувшинов К.Э. и др. Организация мероприятий по профилактике и лечению заболеваний органов дыхания у военнослужащих. Военномедицинский журнал. 2013; 334 (10): 21-44.

5. Сердюков Д.Ю., Гордиенко А.В., Козлов М. и др. Особенности заболеваемости внебольничной пневмонией среди молодого пополнения. Военно-медицинский журнал. 2015; 336 (10): 11-14.

6. Epler G.R., McLoud T.C., Gaensler E.A. et al. Normal chest roentgenograms in chronic diffuse infiltrative lung disease. N. Engl. J. Med. 1978; 298: 934-939.

7. Pellegrino R., Viegi G., Brusasco V. et al. Interpretative strategies for lung function tests. Eur. Respir. J. 2005; 26: 948-968.

8. Wanger J., Clausen J.L., Coates A. et al. Standardisation of the measurement of lung volumes. Eur. Respir. J. 2005; 26: 511-522.

9. Macintyre N., Crapo R.O., Viegi G. et al. Standardisation of the single-breath determination of carbon monoxide uptake in the lung. Eur. Respir. J. 2005; 26: 720-735.

10. Шик Л.Л., Канаев Н.Н., ред. Руководство по клинической физиологии дыхания. Ленинград: Медицина; 1980.

11. Илькович М.М., Кокосов А.Н., ред. Интерстициальные заболевания легких: Руководство для врачей. Раздел «Исследование функции внешнего дыхания». СПб: Нордмедиздат; 2005: 50-59.

12. Owens M.W., Kinasewitz G.T., Anderson W.M. Clinical significance of an isolated reduction in residual volume. Am. Rev. Respir. Dis. 1987; 136: 1377-1380.

13. Воробьева 3.В. Исследование вентиляционной функции легких. М.: Книга и бизнес; 2008.

14. Кольцун С.С. Методы определения остаточного объема легких. Функциональная диагностика. 2003; 1: 65-76.

15. Каменева М.Ю., Тишков А.В., Трофимов В.И. Нерешенные вопросы диагностики рестриктивного типа вентиляционных нарушений. Пульмонология. 2015; 25 (3): 363-367.

Поступила 02.02.16 удк 616.24-002-092

\section{References}

1. Chuchalin A.G., ed. Pulmonology: National Handbook. Moscow: GEOTAR-Media; 2013 (in Russian).

2. Zhogolev S.D., Ogarkov P.I., Zhogolev K.D. et al. Epidemiology and prevention of community-acquired pneumonia in militaries. Voenno-meditsinskiy zhurnal. 2013; 334 (11): 55-60 (in Russian).

3. Kalmykov A.A., Nosarev V.G., Aminev R.M., Konovalov P.P. A role of collaboration between military command and medical service for acute respiratory disease prevention. Voenno-meditsinskiy zhurnal. 2014; 335 (11): 11-16 (in Russian).

4. Ovchinnikov Yu.V., Azarov I.I., Kuvshinov K.E. et al. Management of prevention and treatment of respiratory diseases in militaries. Voenno-meditsinskiy zhurnal. 2013; 334 (10): 21-44 (in Russian).

5. Serdyukov D.Yu., Gordienko A.V., Kozlov M. et al. Particularities of morbidity of community-acquired pneumonia in recruits. Voenno-meditsinskiy zhurnal. 2015; 336 (10): 11-14 (in Russian).

6. Epler G.R., McLoud T.C., Gaensler E.A. et al. Normal chest roentgenograms in chronic diffuse infiltrative lung disease. N. Engl. J. Med. 1978; 298: 934-939.

7. Pellegrino R., Viegi G., Brusasco V. et al. Interpretative strategies for lung function tests. Eur. Respir. J. 2005; 26: 948-968.

8. Wanger J., Clausen J.L., Coates A. et al. Standardisation of the measurement of lung volumes. Eur. Respir. J. 2005; 26: 511-522.

9. Macintyre N., Crapo R.O., Viegi G. et al. Standardisation of the single-breath determination of carbon monoxide uptake in the lung. Eur. Respir. J. 2005; 26: 720-735.

10. Shik L.L., Kanaev N.N., eds. Handbook on Clinical Physiology of Respiration. Leningrad: Meditsina; 1980 (in Russian).

11. Il'kovich M.M., Kokosov A.N., eds. Interstitial Lung Diseases. A Practical Handbook. Chapter "Lung Function Testing". Saint-Petersburg: Nordmedizdat; 2005: 50-59 (in Russian).

12. Owens M.W., Kinasewitz G.T., Anderson W.M. Clinical significance of an isolated reduction in residual volume. Am. Rev. Respir. Dis. 1987; 136: 1377-1380.

13. Vorob'eva Z.V. Testing of Pulmonary Ventilation. Moscow: Kniga i biznes; 2008 (in Russian).

14. Kol'tsun S.S. Methods for measurement of the residual volume of the lungs. Funktsional'naya diagnostika. 2003; 1: 65-76 (in Russian).

15. Kameneva M.Yu., Tishkov A.V., Trofimov V.I. Non-resolved issues of diagnosis of restrictive ventilation disorders. Pul'monologiya. 2015; 25 (3): 363-367 (in Russian).

Received February 02, 2016 UDC 616.24-002-092

\section{Информация об авторах}

Савушкина Ольга Игоревна - к. б. н., зав. отделением функции внешнего дыхания Центра функционально-диагностических исследований ФГКУ «Главный военный клинический госпиталь им. акад. Н.Н.Бурденко» Минобороны России; тел.: (499) 263-55-61; e-mail: olga-savushkina@yandex.ru Черняк Александр Владимирович - К. М. Н., зав. лабораторией функциональных и ультразвуковых методов исследования ФГБУ «НИИ пульмонологии» ФМБА России; тел.: (917) 550-06-34; e-mail: fchi2000 @mail.ru Коповая Наталья Юрьевна - врач 30-го пульмонологического отделения ФГКУ «Главный военный клинический госпиталь им. акад. Н.Н.Бурденко» Минобороны России; тел.: (499) 263-56-30; e-mail: nkopovay @yandex.ru

\section{Author information}

Savushkina Ol'ga Igorevna, PhD, Head of Lung Function Department at Center of Functional Diagnostic Investigations, Acad. N.N.Burdenko The Main Military Clinical Hospital, Moscow, Russia; tel.: (499) 263-55-61; e-mail: olga-savushkina@yandex.ru

Chernyak Aleksandr Vladimirovich, PhD, Head of Laboratory of Functional and Ultra-sound Investigations; Federal Pulmonology Research Institute, Federal Medical and Biological Agency of Russia; tel.: (917) 550-06-34; e-mail: fchi2000@mail.ru

Kopovaya Natal'ya Yur'evna, physician of the $30^{\text {th }}$ Pulmonology Division, Acad. N.N.Burdenko The Main Military Clinical Hospital, Moscow, Russia; tel.: (499) 263-56-30; e-mail: nkopovay@yandex.ru 